\title{
THE SIMANCA METRIC ADMITS A REGULAR QUANTIZATION
}

\author{
FRANCESCO CANNAS AGHEDU, ANDREA LOI
}

\begin{abstract}
Let $g_{S}$ be the Simanca metric on the blow-up $\tilde{\mathbb{C}}^{2}$ of $\mathbb{C}^{2}$ at the origin. We show that $\left(\tilde{\mathbb{C}}^{2}, g_{S}\right)$ admits a regular quantization. We use this fact to prove that all coefficients in the Tian-Yau-Zelditch expansion for the Simanca metric vanish and that a dense subset of $\left(\tilde{\mathbb{C}}^{2}, g_{S}\right)$ admits a Berezin quantization.
\end{abstract}

\section{Contents}

1. Introduction

2. The Simanca metric and the proof of Theorem 1.1

3. Berezin quantization and the proof of Corollary 1.3

4. Balanced metrics on the blow-up of $\mathbb{C}^{n}$ at the origin and the proof of

Theorem 1.4

2010 Mathematics Subject Classification. 53C55; 58C25; 58F06.

Key words and phrases. Kähler manifolds; TYZ asymptotic expansion; Radial metrics; Scalar flat metrics; Projectively induced metrics; Simanca metric; Berezin quantization.

The second author was supported by Prin 2015 - Real and Complex Manifolds; Geometry, Topology and Harmonic Analysis - Italy by INdAM. GNSAGA - Gruppo Nazionale per le Strutture Algebriche, Geometriche e le loro Applicazioni and by Fondazione di Sardegna and Regione Autonoma della Sardegna. 


\section{IntroduCtion}

Let $M$ be a $n$-dimensional complex manifold endowed with a Kähler metric $g$. Assume that there exists a holomorphic line bundle $L$ over $M$ such that $c_{1}(L)=[\omega]$, where $\omega$ is the Kähler form associated to $g$ and $c_{1}(L)$ denotes the first Chern class of $L$. A necessary and sufficient condition for the existence of such an $L$ is that $\omega$ is an integral Kähler form.

Let $m \geq 1$ be an integer and let $h_{m}$ be an Hermitian metric on $L^{m}=L^{\otimes m}$ such that its Ricci curvature $\operatorname{Ric}\left(h_{m}\right)=m \omega$. Here $\operatorname{Ric}\left(h_{m}\right)$ is the two-form on $M$ whose local expression is given by

$$
\operatorname{Ric}\left(h_{m}\right)=-\frac{i}{2 \pi} \partial \bar{\partial} \log h_{m}(\sigma(x), \sigma(x))
$$

for a trivializing holomorphic section $\sigma: U \rightarrow L^{m} \backslash\{0\}$. In the quantum mechanics terminology $L^{m}$ is called the prequantum line bundle, the pair $\left(L^{m}, h_{m}\right)$ is called a geometric quantization of the Kähler manifold $(M, m \omega)$ and $\hbar=m^{-1}$ plays the role of Planck's constant (see e.g. [2]). Consider the separable complex Hilbert space $\mathcal{H}_{m}$ consisting of global holomorphic sections $s$ of $L^{m}$ such that

$$
\langle s, s\rangle_{h_{m}}=\int_{M} h_{m}(s(x), s(x)) \frac{\omega^{n}}{n !}<\infty .
$$

Define

$$
\epsilon_{m g}(x)=\sum_{j=0}^{d_{m}} h_{m}\left(s_{j}(x), s_{j}(x)\right),
$$

where $s_{j}, j=0, \ldots, d_{m}\left(\operatorname{dim} \mathcal{H}_{m}=d_{m}+1 \leq \infty\right)$ is an orthonormal basis of $\left(\mathcal{H}_{m},\langle\cdot, \cdot\rangle_{h_{m}}\right)$. As suggested by the notation this function depends only on the metric $m g$ and not on the orthonormal basis chosen or on the Hermitian metric $h_{m}$. Obviously if $M$ is compact $\mathcal{H}_{m}=H^{0}\left(L^{m}\right)$, where $H^{0}\left(L^{m}\right)$ is the (finite dimensional) space of global holomorphic section of $L^{m}$.

In the literature the function $\epsilon_{m g}$ was first introduced under the name of $\eta$ function by Rawnsley in [33] later renamed as $\theta$-function in [9]. A metric $g$ on $M$ is called balanced if $\epsilon_{g}$ is a positive constant. The definition of balanced metrics was originally given by Donaldson [13] in the case of compact polarized Kähler manifold $(M, g)$ and generalized in [3] to the noncompact case (see also [15], [17], [27]).

A geometric quantization $\left(L^{m}, h_{m}\right)$ of a Kähler manifold $(M, \omega)$ is called a regular quantization $^{1}$ if $m g$ is balanced for any (sufficiently large) natural number $m$.

Many authors (see, e.g. [2] and [5] and references therein) have tried to understand what kind of properties are enjoyed by those Kähler manifolds which admit a regular quantization. Here we recall the following two facts:

\footnotetext{
${ }^{1}$ Regular quantizations were introduced and studied in [9] in the context of quantization by deformation of Kähler manifolds.
} 
- a Kähler metric which admits a regular quantization is cscK (constant scalar curvature Kähler) metric (see [21]);

- a geometric quantization of a homogeneous ${ }^{2}$ and simply-connected Kähler manifold is regular (see [2] and [24] for a proof in the compact and noncompact case respectively).

Therefore, the following question naturally arises: Is it true that a complete ${ }^{3}$ Kähler manifold $(M, \omega)$ which admits a regular quantization is necessarily homogeneous (and simply-connected) ${ }^{4}$ ?

In the compact case this question is still open and of great interest also because the Kähler manifolds involved are projectively algebraic.

In this paper we give a negative answer to the question in the non-compact case by considering the Simanca metric $g_{S}$ on the blow-up $\tilde{\mathbb{C}}^{2}$ of $\mathbb{C}^{2}$ at the origin. The Simanca metric is a well-known and important example (both from mathematical and physical point of view) of non homogeneous complete, zero constant scalar curvature metric (see Section 2 below for details).

Our main result is then the following:

Theorem 1.1. Let $\tilde{\mathbb{C}}^{2}$ be the blow-up of $\mathbb{C}^{2}$ at the origin endowed with the Simanca metric $g_{S}$. Then $\left(\tilde{\mathbb{C}}^{2}, g_{S}\right)$ admits a regular quantization such that $\epsilon_{m g_{S}}=m^{2}$.

It is worth pointing out that recently Bi-Feng-Tu [8] have constructed examples of regular quantizations on Fock-Bargmann-Hartogs domains in the complex Euclidean space equipped with a negative constant scalar curvature Kähler metric. Thus, they provide a negative answer to the previous question in the non-compact case when the scalar curvature is negative. Another important difference between Bi-Feng-Tu example and the Simanca metric is that in the first case the quantization bundle is trivial and the Kähler metric has a global Kähler potential. Moreover, the Simanca metric has been a fundamental ingredient in the construction of cscK metrics on compact Kähler manifold via blow-up procedures (see [6]). Thus we believe our Theorem 1.1 could be used to built regular quantizations of non-homogeneous compact Kähler manifolds.

In order to obtain an interesting corollary of Theorem 1.1 (see Corollary 1.2 below) we need to briefly recall some important tools about asympotic expansions

${ }^{2}$ A Kähler manifold $(M, g)$ is homogeneous if the group $\operatorname{Aut}(M) \cap \operatorname{Isom}(M, g)$ acts transitively on $M$, where $\operatorname{Aut}(M)$ denotes the group of holomorphic diffeomorphisms of $M$ and $\operatorname{Isom}(M, g)$ the isometry group of $(M, g)$.

${ }^{3}$ The assumption of completeness is necessary otherwise one can construct regular quantizations on non-homogeneous Kähler manifolds obtained by deleting a measure zero set from a homogeneous Kähler manifold (see [25]).

${ }^{4}$ The simply-connected request is in brackets since one can prove that every homogeneous and projectively induced Kähler manifold is simply-connected (see [12]). 
of the epsilon function. In the case of a compact Kähler manifold $(M, \omega)$ Zelditch [37] proved that there exists a complete asymptotic expansion in the $C^{\infty}$ category of epsilon function:

$$
\epsilon_{m g}(x) \sim \sum_{j=0}^{\infty} a_{j}(x) m^{n-j}
$$

where $a_{0}(x)=1$ and $a_{j}(x), j=1, \ldots$ are smooth functions on $M$. The expansion (1) is called Tian-Yau-Zelditch expansion (TYZ in the sequel). Later on, Lu [30], by means of Tian's peak section method, proved that each of the coefficients $a_{j}(x)$ is a polynomial of the curvature and its covariant derivatives at $x$ of the metric $g$ which can be found by finitely many algebraic operations. In particular, he computed the first three coefficients. The expression of the first two coefficients is:

$$
\left\{\begin{array}{l}
a_{1}(x)=\frac{1}{2} \rho \\
a_{2}(x)=\frac{1}{3} \Delta \rho+\frac{1}{24}\left(|R|^{2}-4|\operatorname{Ric}|^{2}+3 \rho^{2}\right)
\end{array},\right.
$$

where $\rho, R$, Ric denote respectively the scalar curvature, the curvature tensor and the Ricci tensor of $(M, g)$. The reader is also referred to [19] and [20] for a recursive formula for the coefficients $a_{j}$ 's and an alternative computation of $a_{j}$ for $j \leq 3$ using Calabi's diastasis function (see also [35] for a graph-theoretic interpretation of this recursive formula).

It is natural to study metrics with the Tian-Yau-Zelditch coefficients being prescribed both in the compact that in the noncompact cases. For instance, the vanishing of this coefficients for large enough indexes turns out to be related to some important problems in the theory of pseudoconvex manifolds (cf. [31]). Furthermore, in the noncompact case, one can find in [28] a characterization of the flat metric as a Taub-NUT metric with $a_{3}=0$, while Z. Feng and Z. Tu [16] solve a conjecture formulated in [36] by showing that the complex hyperbolic space is the only Cartan-Hartogs domain where the coefficient $a_{2}$ is constant. In [29] A. Loi and M. Zedda prove that a locally hermitian symmetric space with vanishing $a_{1}$ and $a_{2}$ is flat.

For the Simanca metric $\left(\tilde{\mathbb{C}}^{2}, g_{S}\right)$ in [29] the authors computed the $a_{2}$ coefficient ( $a_{1}=0$ since $g_{S}$ has vanishing scalar curvature). Moreover, in [26] is proved that a projectively induced $^{5}$ radial Kähler metric with $a_{1}=a_{3}=0$ (or with $a_{1}=a_{2}=0$ ) is either the flat metric $g_{0}$ or the Simanca metric $g_{S}$.

Here, by using Theorem 1.1 we immediatly obtain the following:

\footnotetext{
${ }^{5}$ A Kähler metric $g$ on a complex manifold $M$ is said to be projectively induced if exists a holomorphic and isometric (i.e. Kähler) immersion of $(M, g)$ into the complex projective space $\left(\mathbb{C} P^{N}, g_{F S}\right), N \leq+\infty$, endowed with the Fubini-Study metric $g_{F S}$, the metric whose associated Kähler form is given in homogeneous coordinates by $\omega_{F S}=\frac{i}{2 \pi} \partial \bar{\partial} \log \left(\left|Z_{0}\right|^{2}+\cdots+\left|Z_{N}\right|^{2}\right)$.
} 
Corollary 1.2. All the coefficients $a_{j}(x)$, with $j \geq 1$, of the TYZ expansion for the Simanca metric vanish.

Theorem 1.1 shows that $\left(\mathbb{C}^{2}, g_{0}\right)$ and $\left(\tilde{\mathbb{C}}^{2}, g_{S}\right)$ have the same epsilon functions both equal to $m^{2}$. It could be interesting to find other examples of Kähler manifolds sharing this property and, more generally, to analyze to what extent the TYZ coefficients determine the underlying Kähler manifold (cf. [4] for this last issue).

We also prove a result on Berezin's quantization on the dense subset $\mathbb{C}^{2} \backslash\{0\} \subset \tilde{\mathbb{C}}^{2}$ equipped with the restriction of the Simanca Kähler form $\omega_{S}$ associated to the Simanca metric $g_{S}$. This is expressed by the following corollary.

Corollary 1.3. $\left(\mathbb{C}^{2} \backslash\{0\}, \omega_{S}\right)$ admits a Berezin quantization.

The construction in the proof of Theorem 1.1 stops to work when $\mathbb{C}^{2}$ is replaced by $\mathbb{C}^{n}, n \geq 3$ and the metric $g_{S}$ is replaced by its natural generalization $g_{S(n)}$ on $\tilde{\mathbb{C}}^{n}$ (see Section 4 for details). This is expressed by the following theorem.

Theorem 1.4. Let $\tilde{\mathbb{C}}^{n}$ be the blow-up of $\mathbb{C}^{n}$ at the origin endowed with the generalized Simanca metric $g_{S(n)}$. For any integer $m \geq 1$ the following statements hold

(1) $\left(\tilde{\mathbb{C}}^{n}, m g_{S(n)}\right)$ is projectively induced for any $n \geq 2$,

(2) $m g_{S(n)}$ is not balanced for all $n \geq 3$.

That theorem gives an example of projectively induced Kähler metric $g$ on the blow up of $\mathbb{C}^{n}$ at the origin such that $m g$ is not balanced for any positive integer $m$.

The paper is organized as follows. Section 2 contains basic facts on the Simanca metric and the proof of Theorem 1.1. In Section 3, after recalling the definition of Berezin quantization, we prove Corollary 1.3. Finally in Section 4, after describing the well-known link between balanced and projectively induced metrics, we prove Theorem 1.4.

The authors would like to thank the referee for very helpful remarks and also Michela Zedda for useful discussions.

\section{The Simanca Metric And the Proof of Theorem 1.1}

Let us briefly recall the definition of the blow-up $\tilde{\mathbb{C}}^{2}$ of $\mathbb{C}^{2}$ at the origin as

$$
\tilde{\mathbb{C}}^{2}=\left\{\left(z_{1}, z_{2},\left[t_{1}, t_{2}\right]\right) \in \mathbb{C}^{2} \times \mathbb{C} P^{1}: t_{1} z_{2}-t_{2} z_{1}=0\right\} .
$$


$\tilde{\mathbb{C}}^{2}$ is a closed submanifold of $\mathbb{C}^{2} \times \mathbb{C} P^{1}$ of complex dimension 2. A system of charts for $\tilde{\mathbb{C}}^{2}$ is given as follows: for $j=1,2$ we take

$$
\tilde{U}_{j}=\left(\mathbb{C}^{2} \times U_{j}\right) \cap \tilde{\mathbb{C}}^{2}
$$

where $U_{j}=\left\{t_{j} \neq 0\right\}$, for $j=1,2$, are open subsets of $\mathbb{C} P^{1}$. Then we have two coordinate maps

$$
\begin{aligned}
& \varphi_{1}: \tilde{U}_{1} \rightarrow \mathbb{C}^{2},\left(z_{1}, z_{2},\left[t_{1}, t_{2}\right]\right) \mapsto\left(z_{1}, \frac{t_{2}}{t_{1}}\right), \\
& \varphi_{2}: \tilde{U}_{2} \rightarrow \mathbb{C}^{2},\left(z_{1}, z_{2},\left[t_{1}, t_{2}\right]\right) \mapsto\left(\frac{t_{1}}{t_{2}}, z_{2}\right),
\end{aligned}
$$

having as inverses the parametrization maps defined, respectively, by

$$
\begin{aligned}
& \varphi_{1}^{-1}: \mathbb{C}^{2} \rightarrow \tilde{U}_{1},\left(w_{1}, w_{2}\right) \mapsto\left(w_{1}, w_{1} w_{2},\left[1, w_{2}\right]\right), \\
& \varphi_{2}^{-1}: \mathbb{C}^{2} \rightarrow \tilde{U}_{2},\left(w_{1}, w_{2}\right) \mapsto\left(w_{1} w_{2}, w_{2},\left[w_{1}, 1\right]\right) .
\end{aligned}
$$

There are two projection maps

$$
\begin{aligned}
& p_{1}: \tilde{\mathbb{C}}^{2} \rightarrow \mathbb{C}^{2}, \\
& p_{2}: \tilde{\mathbb{C}}^{2} \rightarrow \mathbb{C} P^{1},
\end{aligned}
$$

given by the restriction to $\tilde{\mathbb{C}}^{2}$ of the canonical projections of $\mathbb{C}^{2} \times \mathbb{C} P^{1}$. One can prove (see [32]) that $p_{2}$ induces on $\tilde{\mathbb{C}}^{2}$ the structure of complex line bundle, whose fibre over $\left[t_{1}, t_{2}\right] \in \mathbb{C} P^{1}$ is the corresponding line $\left\{\left(\lambda t_{1}, \lambda t_{2}\right) \mid \lambda \in \mathbb{C}\right\}$ in $\mathbb{C}^{2}$. In other words, this is the universal line bundle over $\mathbb{C} P^{1}$. Observe that $p_{1}$ is bijective when restricted to $p_{1}^{-1}\left(\mathbb{C}^{2} \backslash\{0\}\right)$, while

$$
p_{1}^{-1}(0)=\left\{(0,[t]) \in \tilde{\mathbb{C}}^{2}\right\} \simeq \mathbb{C} P^{1} .
$$

Thus we may think of $\tilde{\mathbb{C}}^{2}$ as obtained from $\mathbb{C}^{2}$ by replacing the origin 0 by the space of all lines in $\mathbb{C}^{2}$ through 0 . The manifold $p_{1}^{-1}(0)$ is called the exceptional divisor, and we will denote it by $H$. So, the restriction

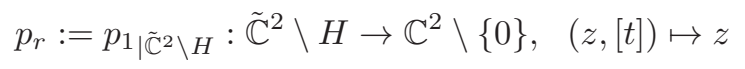

is a biholomorphism, having as inverse

$$
\mathbb{C}^{2} \backslash\{0\} \rightarrow \tilde{\mathbb{C}}^{2} \backslash H, \quad z \mapsto\left(z_{1}, z_{2},\left[z_{1}, z_{2}\right]\right) .
$$

Take now on $\mathbb{C}^{2} \backslash\{0\}$ the $(1,1)$-form given by

$$
\omega=\frac{i}{2 \pi} \partial \bar{\partial}\left(|z|^{2}+\log |z|^{2}\right) .
$$

We claim that the pull-back $p_{r}^{*}(\omega)$ of $\omega$, a priori defined only on $\tilde{\mathbb{C}}^{2} \backslash H$, extends in fact to all $\tilde{\mathbb{C}}^{2}$. 
The pull-back $p_{r}^{*}(\omega)$ is given in the coordinates (3) by

$$
p_{r}^{*}(\omega)=\frac{i}{2 \pi} \partial \bar{\partial}\left(\left|z_{1}\right|^{2}\left(1+\left|z_{2}\right|^{2}\right)+\log \left(1+\left|z_{2}\right|^{2}\right)\right),
$$

on $\tilde{U}_{1} \backslash H$, and

$$
p_{r}^{*}(\omega)=\frac{i}{2 \pi} \partial \bar{\partial}\left(\left|z_{2}\right|^{2}\left(1+\left|z_{1}\right|^{2}\right)+\log \left(1+\left|z_{1}\right|^{2}\right)\right),
$$

on $\tilde{U}_{2} \backslash H$. This shows that $p_{r}^{*}(\omega)$ extends to the whole $\tilde{\mathbb{C}}^{2}$, as claimed. Clearly on $\tilde{\mathbb{C}}^{2} \backslash H$ this form is given in local coordinates by (4). The metric associated to (4) was introduced for the first time by S. Simanca in [34] and it is known in literature as the Simanca metric and denoted here by $g_{S}$. The form (4) is denoted here by $\omega_{S}$. It is not hard to see that $g_{S}$ is a complete, zero constant scalar curvature Kähler metric ([34]). Moreover $\left(\tilde{\mathbb{C}}^{2}, \omega_{S}\right)$ is a non homogeneous manifold (see, e.g. [29]). Notice also that $\tilde{\mathbb{C}}^{2}$ is non contractible but simply-connected since $\tilde{\mathbb{C}}^{2}$ is diffeomorphic to the connected sum $\mathbb{C}^{2} \# \overline{\mathbb{C} P^{2}}$, where $\overline{\mathbb{C} P^{2}}$ is the complex projective space $\mathbb{C} P^{2}$ with the opposite orientation.

In order to prove Theorem 1.1 consider the holomorphic line bundle $L \rightarrow \tilde{\mathbb{C}}^{2}$ such that $c_{1}(L)=\left[\omega_{S}\right]$, where $c_{1}(L)$ is the first Chern class of $L$. Such line bundle exists since $\omega_{S}$ is integral and it is unique, up to isomorphisms of line bundle, since $\tilde{\mathbb{C}}^{2}$ is simply-connected. Notice that this line bundle $L$ is not trivial and the Kähler form $\omega_{S}$ associated to $g_{S}$ does not admit a global Kähler potential in contrast with the example of Bi-Feng-Tu discussed in the introduction.

One can easily verify that the holomorphic line bundle $L^{m} \rightarrow \tilde{\mathbb{C}}^{2}$ equipped with the hermitian structure

$$
h_{m}(\sigma(x), \sigma(x))=\frac{1}{|z|^{2 m}} e^{-m|z|^{2}}|q|^{2},
$$

defines a geometric quantization of $\left(\tilde{\mathbb{C}}^{2}, m \omega_{S}\right)$, where $m$ is a positive natural number and $\sigma: U \subset \tilde{\mathbb{C}}^{2} \backslash H \rightarrow L^{m} \backslash\{0\}, x \mapsto(z, q) \in U \times \mathbb{C}$ is a trivialising holomorphic section. Since $L_{\mid \mathbb{C}^{2} \backslash\{0\}}^{m}$ is equivalent to the trivial bundle $\mathbb{C}^{2} \backslash\{0\} \times \mathbb{C}$, one can find a natural bijection between the complex space $H^{0}\left(L^{m}\right)$ and the space of holomorphic functions on $\mathbb{C}^{2}$ vanishing at the origin with order greater or equal than $m$ (see, e.g. [18, Chapter 1]). This bijection takes $s \in H^{0}\left(L^{m}\right)$ to the holomorphic function $f_{s}$ on $\mathbb{C}^{2}$ obtained by restricting $s$ to $\tilde{\mathbb{C}}^{2} \backslash H \simeq \mathbb{C}^{2} \backslash\{0\}$. Moreover, since $H$ has zero measure in $\tilde{\mathbb{C}}^{2}$, one gets

$$
\begin{aligned}
\langle s, s\rangle_{h_{m}} & =\int_{\tilde{\mathbb{C}}^{2}} h_{m}(s(x), s(x)) \frac{\omega_{S}^{2}}{2 !}= \\
& =\int_{\mathbb{C}^{2} \backslash\{0\}} \frac{e^{-m|z|^{2}}}{|z|^{2 m}}\left|f_{s}(z)\right|^{2}\left(1+\frac{1}{|z|^{2}}\right) d \mu(z)<\infty,
\end{aligned}
$$


where $d \mu(z)=\left(\frac{i}{2 \pi}\right)^{2} d z_{1} \wedge d \bar{z}_{1} \wedge d z_{2} \wedge d \bar{z}_{2}$. Therefore, in this case, the inclusion $\mathcal{H}_{m} \subseteq H^{0}\left(L^{m}\right)$ is indeed an equality, namely $\mathcal{H}_{m}=H^{0}\left(L^{m}\right)^{6}$.

We are now ready to prove Theorem 1.1.

Proof of Theorem 1.1. By passing to polar coordinates $z_{1}=\rho_{1} e^{i \vartheta_{1}}, z_{2}=\rho_{2} e^{i \vartheta_{2}}$ with $\rho_{1}, \rho_{2} \in(0,+\infty), \vartheta_{1}, \vartheta_{2} \in(0,2 \pi)$ one easily sees that the monomials $\left\{z_{1}^{j} z_{2}^{k}\right\}_{j+k \geq m}$ form a complete orthogonal system for the Hilbert space $\left(\mathcal{H}_{m},\langle\cdot, \cdot\rangle_{h_{m}}\right)$. Moreover, by (5),

$$
\left\|z_{1}^{j} z_{2}^{k}\right\|_{h_{m}}^{2}=4 \int_{0}^{+\infty} \int_{0}^{+\infty} \frac{e^{-m\left(\rho_{1}^{2}+\rho_{2}^{2}\right)}}{\left(\rho_{1}^{2}+\rho_{2}^{2}\right)^{m+1}}\left(1+\rho_{1}^{2}+\rho_{2}^{2}\right) \rho_{1}^{2 j} \rho_{2}^{2 k} \rho_{1} \rho_{2} d \rho_{1} d \rho_{2} .
$$

With the substitution $\rho_{1}=r \cos \theta, \rho_{2}=r \sin \theta, 0<r<+\infty, 0<\theta<\frac{\pi}{2}$ one finds a product of one variable integral:

$$
\left\|z_{1}^{j} z_{2}^{k}\right\|_{h_{m}}^{2}=4 \int_{0}^{\frac{\pi}{2}}(\cos \theta)^{2 j+1}(\sin \theta)^{2 k+1} d \theta \cdot \int_{0}^{+\infty} r^{2(j+k-m)+1}\left(1+r^{2}\right) e^{-m r^{2}} d r .
$$

For the first integral we have (see [1, page 255 (6.1.1)])

$$
\int_{0}^{\frac{\pi}{2}}(\cos \theta)^{2 j+1}(\sin \theta)^{2 k+1} d \theta=\frac{\Gamma(j+1) \Gamma(k+1)}{2 \Gamma(j+k+2)}=\frac{j ! k !}{2(j+k+1) !} .
$$

For the second intergral, by (see [1, page $258(6.2 .1)]$ )

$$
\int_{0}^{\infty} r^{s} e^{-m r^{2}} d r=\frac{\Gamma\left(\frac{s+1}{2}\right)}{2 m^{\left(\frac{s+1}{2}\right)}}
$$

we find

$$
\begin{aligned}
\int_{0}^{\infty} r^{2(j+k-m)+1}\left(1+r^{2}\right) e^{-m r^{2}} d r & =\frac{\Gamma(j+k-m+1)}{2 m^{j+k-m+1}}+\frac{\Gamma(j+k-m+2)}{2 m^{j+k-m+2}}= \\
& =\frac{(j+k-m) !(j+k+1)}{2 m^{j+k-m+2}} .
\end{aligned}
$$

Hence one gets

$$
\left\|z_{1}^{j} z_{2}^{k}\right\|_{h_{m}}^{2}=4\left[\frac{j ! k !}{2(j+k+1) !}\right] \cdot\left[\frac{(j+k-m) !(j+k+1)}{2 m^{j+k-m+2}}\right]=\frac{j ! k !}{(j+k) !} \frac{(j+k-m) !}{m^{j+k-m+2}} .
$$

Therefore

$$
\left\{\frac{z_{1}^{j} z_{2}^{k}}{\left\|z_{1}^{j} z_{2}^{k}\right\|_{h_{m}}}\right\}_{j+k \geq m}
$$

is an orthonormal basis for the Hilber Space $\left(\mathcal{H}_{m},\langle\cdot, \cdot\rangle_{h_{m}}\right)$.

\footnotetext{
${ }^{6}$ Here $\mathcal{H}_{m}$ (as in the introduction) denotes the space of global holomorphic sections $s$ of $L^{m}$, which are bounded with respect to

$$
\langle s, s\rangle_{h_{m}}=\|s\|_{h_{m}}^{2}=\int_{\tilde{\mathbb{C}}^{2}} h_{m}(s(x), s(x)) \frac{\omega_{S}^{2}}{2 !} .
$$
}


For the epsilon function one obtains

$$
\begin{aligned}
\epsilon_{m g}(z) & =\sum_{\substack{j, k \geq 0 \\
j+k \geq m}} \frac{e^{-m\left(\left|z_{1}\right|^{2}+\left|z_{2}\right|^{2}\right)}}{\left(\left|z_{1}\right|^{2}+\left|z_{2}\right|^{2}\right)^{m}} \frac{\left|z_{1}\right|^{2 j}\left|z_{2}\right|^{2 k}}{|| z_{1}^{j} z_{2}^{k}||_{h_{m}}^{2}}= \\
& =\frac{e^{-m\left(\left|z_{1}\right|^{2}+\left|z_{2}\right|^{2}\right)}}{\left(\left|z_{1}\right|^{2}+\left|z_{2}\right|^{2}\right)^{m}} \sum_{\substack{j, k \geq 0 \\
j+k \geq m}} \frac{(j+k) !\left|z_{1}\right|^{2 j}\left|z_{2}\right|^{2 k}}{j ! k !(j+k-m) !} m^{j+k-m+2}= \\
& =\frac{e^{-m\left(\left|z_{1}\right|^{2}+\left|z_{2}\right|^{2}\right)}}{\left(\left|z_{1}\right|^{2}+\left|z_{2}\right|^{2}\right)^{m}} \sum_{\beta=m}^{\infty}\left[\sum_{j, k \geq 0} \frac{(j+k) !\left|z_{1}\right|^{2 j}\left|z_{2}\right|^{2 k}}{j ! k !}\right] \frac{m^{\beta-m+2}}{(\beta-m) !}= \\
& =\frac{e^{-m\left(\left|z_{1}\right|^{2}+\left|z_{2}\right|^{2}\right)}}{\left(\left|z_{1}\right|^{2}+\left|z_{2}\right|^{2}\right)^{m}} \sum_{\beta=m}^{\infty}\left(\left|z_{1}\right|^{2}+\left|z_{2}\right|^{2}\right)^{\beta} \frac{m^{\beta-m+2}}{(\beta-m) !}= \\
& =m^{2} e^{-m\left(\left|z_{1}\right|^{2}+\left|z_{2}\right|^{2}\right)} \sum_{\beta=m}^{\infty}\left(\left|z_{1}\right|^{2}+\left|z_{2}\right|^{2}\right)^{\beta-m} \frac{m^{\beta-m}}{(\beta-m) !}= \\
& =m^{2} e^{-m\left(\left|z_{1}\right|^{2}+\left|z_{2}\right|^{2}\right)} \sum_{\alpha=0}^{\infty}\left(\left|z_{1}\right|^{2}+\left|z_{2}\right|^{2}\right)^{\alpha} \frac{m^{\alpha}}{\alpha !}= \\
& =m^{2}, \quad
\end{aligned}
$$

and this proves the theorem.

\section{Berezin quantization And the PRoof of Corollary 1.3}

Let $(M, \omega)$ be a symplectic manifold and let $\{\cdot, \cdot\}$ be the associated Poisson bracket. A Berezin quantization (we refer to [7] for details) on $M$ is given by a family of associative algebras $\mathcal{A}_{\hbar}$ where the parameter $\hbar$ (which plays the role of the Planck constant) ranges over a set $E$ of positive reals with limit point 0 . Then in the direct sum $\oplus_{\hbar \in E} \mathcal{A}_{\hbar}$ with component-wise product $*$, there exists a subalgebra $\mathcal{A}$, such that for an arbitrary element $f=f(\hbar) \in A$, where $f(\hbar) \in \mathcal{A}_{\hbar}$, there exists a limit $\lim _{\hbar \rightarrow 0} f(\hbar)=\varphi(f) \in C^{\infty}(M)$. The following correspondence principle must hold: for $f, g \in \mathcal{A}$

$$
\varphi(f * g)=\varphi(f) \varphi(g), \quad \varphi\left(\hbar^{-1}(f * g-g * f)\right)=i\{\varphi(f), \varphi(g)\} .
$$

Moreover, for any pair of points $x_{1}, x_{2} \in M$ there exists $f \in \mathcal{A}$ such that $\varphi(f)\left(x_{1}\right) \neq$ $\varphi(f)\left(x_{2}\right)$.

Consider now a real analytic Kähler manifold $M$, with Kähler metric $g$ and associated Kähler form $\omega$. Assume that there exists a (real analytic) global Kähler potential $\Phi: M \rightarrow \mathbb{R}$. This function extends to a sesquianalytic function $\Phi(x, \bar{y})$ on that neighbourhood of the diagonal in $M \times M$ such that $\Phi(x, \bar{x})=\Phi(x)$. Consider Calabi's diastasis function $D_{g}$ defined on a neighbourhood of the diagonal in $M \times M$ 
by:

$$
D_{g}(x, y)=\Phi(x, \bar{x})+\Phi(y, \bar{y})-\Phi(x, \bar{y})-\Phi(y, \bar{x}) .
$$

By its definition we see that Calabi's diastasis function is independent from the potential chosen which is defined up to the sum with the real part of a holomorphic function. Moreover, it is easily seen that $D_{g}$ is real-valued, symmetric in $x$ and $y$ and $D_{g}(x, x)=0$ (the reader is referred to [10] and [22] for more details on the diastasis function).

Example 3.1. Let $g_{F S}$ be the Fubini-Study metric on the infinite dimensional complex projective space $\mathbb{C} P^{\infty}$ of holomorphic sectional curvature 4 and let $D_{g_{F S}}(p, q)$ be the associated Calabi's diastasis function. One can show that for all $p \in \mathbb{C} P^{\infty}$ the function $D_{g_{F S}}(p, \cdot)$ is globally defined except in the cut locus $H_{p}$ of $p$ where it blows up. Moreover $e^{-D_{g_{F S}}(p, q)}$ is globally defined and smooth on $\mathbb{C} P^{\infty}, e^{-D_{g_{F S}}(p, q)} \leq 1$ and $e^{-D_{g_{F S}}(p, q)}=1$ if and only if $p=q$ (see [22] for details).

The following theorem is a reformulation of Berezin quantization result (see [14] and [23]) in terms of Rawnsley $\epsilon$-function and Calabi's diastasis function.

Theorem 3.2. Let $\Omega \subset \mathbb{C}^{n}$ be a complex domain equipped with a real analytic Kähler form $\omega$ and corresponding Kähler metric g. Then, $(\Omega, \omega)$ admits a Berezin quantization if the following two conditions are satisfied:

(1) Rawnsley's function $\epsilon_{m g}(x)$ is a positive constant for all sufficiently large $m$;

(2) the function $e^{-D_{g}(x, y)}$ is globally defined on $\Omega \times \Omega, e^{-D_{g}(x, y)} \leq 1$ and $e^{-D_{g}(x, q y)}=1$ if and only if $x=y$.

We are now in the position to prove Corollary 1.3, namely that $\left(\mathbb{C}^{2} \backslash\{0\}, \omega_{S}\right)$ admits a Berezin quantization.

Proof of Corollary 1.3. We are going to show that Conditions 1 and 2 of Theorem 3.2 are fulfilled by $\left(\mathbb{C}^{2} \backslash\{0\}, \omega_{S}\right)$. Condition 1 follows by Theorem 1.1. For Condition 2 consider the holomorphic map

$$
\varphi: \mathbb{C}^{2} \backslash\{0\} \rightarrow \mathbb{C} P^{\infty}
$$

given by

$$
\left(z_{1}, z_{2}\right) \mapsto\left(z_{1}, z_{2}, \ldots, \sqrt{\frac{j+k}{j ! k !}} z_{1}^{j} z_{2}^{k}, \ldots\right), j+k \neq 0 .
$$

It is not hard to see that $\varphi$ is an injective Kähler immersion from $\left(\mathbb{C}^{2} \backslash\{0\}, g_{S}\right)$ into $\left(\mathbb{C} P^{\infty}, g_{F S}\right)$ (see [26] for a proof). By Example 3.1, Calabi's diastasis function $D_{g_{F S}}$ of $\mathbb{C} P^{\infty}$ is such that $e^{-D_{g_{F S}}}$ is globally defined on $\mathbb{C} P^{\infty} \times \mathbb{C} P^{\infty}$ and by the hereditary property of the diastasis function (see [10, Proposition 6]) we get that, 
for all $x, y \in \mathbb{C}^{2} \backslash\{0\}$,

$$
e^{-D_{F S}(\varphi(x), \varphi(y))}=e^{-D_{g_{S}}(x, y)}
$$

is globally defined on $\mathbb{C}^{2} \backslash\{0\} \times \mathbb{C}^{2} \backslash\{0\}$. Since, by Example 3.1, $e^{-D_{F S}(p, q)} \leq 1$ for all $p, q \in \mathbb{C} P^{\infty}$ it follows that $e^{-D_{g_{S}}(x, y)} \leq 1$ for all $x, y \in \mathbb{C}^{2} \backslash\{0\}$ and since $\varphi$ is injective one gets that $e^{-D_{g_{S}}(x, y)}=1$ iff $x=y$. Hence, also Condition 2 is satisfied and this concludes the proof of the corollary.

\section{BALANCED MEtrics on the BlOW-UP of $\mathbb{C}^{n}$ AT the ORIGIN AND the PROOF OF THEOREM 1.4}

It is well known (see [9] and [33]) that if $m g$ is a balanced metric, namely the function $\epsilon_{m g}$ is a positive constant, then $m g$ is projectively induced via the coherent states map

$$
\varphi_{m}: M \rightarrow \mathbb{C} P^{d_{m}}, x \mapsto\left[s_{0}(x), \ldots, s_{j}(x), \ldots\right] .
$$

In fact the relation between this map and the function $\epsilon_{m g}$ can be read in the following formula due to Rawnsley (see [33]):

$$
\varphi_{m}^{*}\left(\omega_{F S}\right)=\frac{i}{2 \pi} \partial \bar{\partial} \log \sum_{j=0}^{d_{m}}\left|s_{j}(x)\right|^{2}=m \omega+\frac{i}{2 \pi} \partial \bar{\partial} \log \epsilon_{m g} .
$$

Therefore, by Theorem 1.1 the metric $m g_{S}$ on $\tilde{\mathbb{C}}^{2}$ is projectively induced for all $m>0$. Another example of Kähler metric on the blow-up $\tilde{\mathbb{C}}^{2}$ of $\mathbb{C}^{2}$ at the origin is the celebrated Eguchi-Hanson metric $g_{E H}$, namely the complete Ricci flat metric on $\tilde{\mathbb{C}}^{2}$ whose Kähler form on $\tilde{\mathbb{C}}^{2} \backslash H \cong \mathbb{C}^{2} \backslash\{0\}$ is given by

$$
\omega_{E H}=\frac{i}{2 \pi} \partial \bar{\partial}\left(\sqrt{|z|^{4}+1}+\log |z|^{2}-\log \left(1+\sqrt{|z|^{4}+1}\right) .\right.
$$

Recently in [11], the first author of the present paper shows that the metric $m g_{E H}$ is not balanced for any positive integer $m$. Hence, it is natural to see if there exist examples of complete metrics $g$ on the blow-up of the Euclidean space at the origin such that $m g$ is projectively induced but $m g$ is not balanced for any $m>0$. In this section we construct such a metric.

Let $g_{S(n)}$ be the generalized Simanca metric on the blow-up $\tilde{\mathbb{C}}^{n}$ of $\mathbb{C}^{n}$ at the origin whose Kähler form on $\mathbb{C}^{n} \backslash\{0\} \cong \tilde{\mathbb{C}}^{n} \backslash H$ is given by

$$
\omega_{S(n)}=\frac{i}{2 \pi} \partial \bar{\partial}\left(|z|^{2}+\log |z|^{2}\right), \quad|z|^{2}=\left|z_{1}\right|^{2}+\cdots+\left|z_{n}\right|^{2}
$$

and $H$ denotes the exceptional divisor arising by the blow-up construction (as in Section 2 one can show that $\omega_{S(n)}$, a priori defined only on $\mathbb{C}^{n} \backslash\{0\}$, extends to all $\left.\tilde{\mathbb{C}}^{n}\right)$. When $n=2, g_{S(2)}=g_{S}$. Notice that the generalized Simanca metric is complete but its scalar curvature is not constant. 
Remark 4.1. In the case of the generalized Simanca metric, for $n=3$ we have

$$
g_{S(3)}=\left(\begin{array}{ccc}
1+\frac{\left|z_{2}\right|^{2}+\left|z_{3}\right|^{2}}{\left(\left|z_{1}\right|^{2}+\left|z_{2}\right|^{2}+\left|z_{3}\right|^{2}\right)} & -\frac{z_{1}}{\left(\left|z_{1}\right|^{2}+\left.z_{2} z_{2}\right|^{2}+\left|z_{3}\right|^{2}\right)} & -\frac{\bar{z}_{1} z_{3}}{\left(\left|z_{1}\right|^{2}+\left|z_{2}\right|^{2}+\left|z_{3}\right|^{2}\right)} \\
-\frac{\left.z_{1}\right|^{2}+\left|z_{3}\right|^{2}}{\left(\left|z_{1}\right|^{2}+\left|z_{2}\right|^{2}+\left|z_{3}\right|^{2}\right)} & 1+\frac{z_{1} z_{3}}{\left(\left|z_{1}\right|^{2}\left|z_{2}\right|^{2}+\left|z_{3}\right|^{2}\right)} & -\frac{z_{3}}{\left(\left|z_{1}\right|^{2}+\left|z_{2}\right|^{2}+\left|z_{3}\right|^{2}\right)} \\
-\frac{z_{2}}{\left(\left|z_{1}\right|^{2}+\left|z_{2}\right|^{2}+\left|z_{3}\right|^{2}\right)} & -\frac{\left|z_{1}\right|^{2}+\left|z_{2}\right|^{2}}{\left(\left|z_{1}\right|^{2}+\left|z_{2}\right|^{2}+\left|z_{3}\right|^{2}\right)} & 1+\frac{1+\frac{z_{1}}{\left(\left|z_{1}\right|^{2}+\left|z_{2}\right|^{2}+\left|z_{3}\right|^{2}\right)}}{}
\end{array}\right)
$$

so that, for $\left(z_{1}, 0,0\right)$

$$
g_{S(3)}=\left(\begin{array}{ccc}
1 & 0 & 0 \\
0 & 1+\frac{1}{\left|z_{1}\right|^{2}} & 0 \\
0 & 0 & 1+\frac{1}{\left|z_{1}\right|^{2}}
\end{array}\right), \quad g_{S(3)}^{-1}=\left(\begin{array}{ccc}
1 & 0 & 0 \\
0 & \frac{\left|z_{1}\right|^{2}}{1+\left|z_{1}\right|^{2}} & 0 \\
0 & 0 & \frac{\left|z_{1}\right|^{2}}{1+\left|z_{1}\right|^{2}}
\end{array}\right) \text {. }
$$

By recalling that $R i c_{i \bar{j}}=-\frac{\partial^{2} \log \operatorname{det} g}{\partial z_{i} \partial \bar{z}_{j}}$ one gets:

$$
\operatorname{Ric}=\left(\begin{array}{ccc}
-\frac{1}{\left(1+\left|z_{1}\right|^{2}\right)^{2}} & 0 & 0 \\
0 & \frac{1}{\left|z_{1}\right|^{2}+\left|z_{1}\right|^{4}} & 0 \\
0 & 0 & \frac{1}{\left|z_{1}\right|^{2}+\left|z_{1}\right|^{4}}
\end{array}\right) \text {. }
$$

Finally, by recalling that $\rho_{g}=-\sum g^{i \bar{j}} R i c_{i \bar{j}}$, one finds:

$$
\rho_{g_{S(3)}}=-\frac{1}{\left(1+\left|z_{1}\right|^{2}\right)^{2}} .
$$

More generally it is possible to prove that

$$
\rho_{g_{S(n)}}=\frac{2-n}{\left(1+\left|z_{1}\right|^{2}\right)^{2}}, \quad \text { for } \quad\left(z_{1}, 0, \ldots, 0\right)
$$

so the scalar curvature is not costant for any $n \geq 3$.

Proof of Theorem 1.4. 1. The holomorphic map

$$
\varphi: \mathbb{C}^{n} \backslash\{0\} \rightarrow \mathbb{C} P^{\infty}
$$

given by

$$
\left(z_{1}, \ldots, z_{n}\right) \mapsto\left(z_{1}, \ldots, z_{n}, \ldots, \sqrt{\frac{j_{1}+\cdots+j_{n}}{j_{1} ! \cdots j_{n} !}} z_{1}^{j_{1}} \cdots z_{n}^{j_{n}}, \ldots\right), j_{1}+\cdots+j_{n} \neq 0,
$$

is a Kähler immersion from $\left(\mathbb{C}^{n} \backslash\{0\}, g_{S(n)}\right)$ into $\left(\mathbb{C} P^{\infty}, g_{F S}\right)$. In point of fact

$$
\begin{aligned}
\varphi^{*}\left(\omega_{F S}\right) & =\frac{i}{2 \pi} \partial \bar{\partial} \log \left(\sum_{\substack{j_{1}, j_{2}, \ldots, j_{n} \geq 0 \\
j_{1}+\cdots+j_{n} \geq m}}\left(\frac{j_{1}+\cdots+j_{n}}{j_{1} ! \cdots j_{n} !}\left|z_{1}\right|^{2 j_{1}} \cdots\left|z_{n}\right|^{2 j_{n}}\right)\right)= \\
& =\frac{i}{2 \pi} \partial \bar{\partial} \log \left(e^{|z|^{2}}|z|^{2}\right)=\omega_{S(n)} .
\end{aligned}
$$

Since $\widetilde{\mathbb{C}}^{n}$ is simply-connected it follows from a result of Calabi (see [10] and [26]) that $\varphi$ extends to a Kähler immersion from $\left(\tilde{\mathbb{C}}^{n}, g_{S(n)}\right)$ into $\left(\mathbb{C} P^{\infty}, g_{F S}\right)$. Similarly, one can show that $m g_{S(n)}$ is projectively induced for any positive integer $m$. Indeed, 
by a result of Calabi [10] if a Kähler manifold can be Kähler immersed into $\mathbb{C} P^{\infty}$ then the same is true for $(M, m g)$.

2. For an integer $m>0$, consider the geometric quantization given by the holomorphic line bundle $L^{m} \rightarrow\left(\tilde{\mathbb{C}}^{n}, \omega_{S(n)}\right)$ such that $c_{1}\left(L^{m}\right)=m\left[\omega_{S(n)}\right]$, equipped with the hermitian structure

$$
h_{m}(\sigma(x), \sigma(x))=\frac{1}{|z|^{2 m}} e^{-m|z|^{2}}|q|^{2} .
$$

where $\sigma: U \subset \tilde{\mathbb{C}}^{n} \backslash H \rightarrow L^{m} \backslash\{0\}, x \mapsto(z, q) \in U \times \mathbb{C}$ is a trivialising holomorphic section. As for the Simanca metric there is a natural bijection between the complex space $H^{0}\left(L^{m}\right)$ of global holomorphic sections and the space of holomorphic functions on $\mathbb{C}^{n}$ vanishing at the origin with order greater or equal than $m$. This bijection takes $s \in H^{0}\left(L^{m}\right)$ to the holomorphic function $f_{s}$ on $\mathbb{C}^{n}$ obtained by restricting $s$ to $\tilde{\mathbb{C}}^{n} \backslash H \simeq \mathbb{C}^{n} \backslash\{0\}$. Moreover, since $H$ has zero measure in $\tilde{\mathbb{C}}^{n}$, one gets

$$
\begin{aligned}
\langle s, s\rangle_{h_{m}} & =\int_{\tilde{\mathbb{C}}^{n}} h_{m}(s(x), s(x)) \frac{\omega_{S(n)}^{n}}{n !}= \\
& =\int_{\mathbb{C}^{n} \backslash\{0\}} \frac{e^{-m|z|^{2}}}{|z|^{2 m}}\left|f_{s}(z)\right|^{2}\left(1+\frac{1}{|z|^{2}}\right)^{n-1} d \mu(z)<\infty,
\end{aligned}
$$

where $d \mu(z)=\left(\frac{i}{2 \pi}\right)^{n} d z_{1} \wedge d \bar{z}_{1} \wedge d z_{2} \wedge d \bar{z}_{2} \wedge \ldots \wedge d z_{n} \wedge d \bar{z}_{n}$. Therefore $\mathcal{H}_{m}=H^{0}\left(L^{m}\right)$.

From (12) by passing to polar coordinates $z_{1}=\rho_{1} e^{i \vartheta_{1}}, \ldots, z_{n}=\rho_{n} e^{i \vartheta_{n}}$ with $\rho_{1}, \ldots \rho_{n} \in(0,+\infty), \vartheta_{1}, \ldots, \vartheta_{n} \in(0,2 \pi)$ one easily sees that the set

$$
\left\{z_{1}^{j_{1}} \cdots z_{n}^{j_{n}}\right\}, \quad j_{1}+\cdots+j_{n} \geq m
$$

form a complete orthogonal system for the Hilbert space $\left(\mathcal{H}_{m},\langle\cdot, \cdot\rangle_{h_{m}}\right)$. Moreover, by (12),

$\left\|z_{1}^{j_{1}} \cdots z_{n}^{j_{n}}\right\|_{h_{m}}^{2}=2^{n} \int_{\Omega} \frac{e^{-m\left(\rho_{1}^{2}+\cdots+\rho_{n}^{2}\right)}}{\left(\rho_{1}^{2}+\cdots+\rho_{n}^{2}\right)^{m+n-1}}\left(1+\rho_{1}^{2}+\cdots+\rho_{n}^{2}\right)^{n-1} \rho_{1}^{2 j_{1}+1} \cdots \rho_{n}^{2 j_{n}+1} d \rho_{1} \cdots d \rho_{n}$,

where $\Omega=\left\{(0,+\infty)^{n} \subset \mathbb{R}^{n}\right\}$. With the substitution

$$
\begin{aligned}
& \rho_{1}=r \cos \left(\vartheta_{1}\right) \\
& \rho_{2}=r \sin \left(\vartheta_{1}\right) \cos \left(\vartheta_{2}\right) \\
& \rho_{3}=r \sin \left(\vartheta_{1}\right) \sin \left(\vartheta_{2}\right) \cos \left(\vartheta_{3}\right) \\
& \vdots \\
& \rho_{n-1}=r \sin \left(\vartheta_{1}\right) \cdots \sin \left(\vartheta_{n-2}\right) \cos \left(\vartheta_{n-1}\right) \\
& \rho_{n}=r \sin \left(\vartheta_{1}\right) \cdots \sin \left(\vartheta_{n-2}\right) \sin \left(\vartheta_{n-1}\right)
\end{aligned}
$$


with $0<r<+\infty, 0<\vartheta_{i}<\frac{\pi}{2}$ for $i=1, \ldots, n-1$, one finds a product of one variable integrals:

$$
\begin{aligned}
\left\|z_{1}^{j_{1}} \cdots z_{n}^{j_{n}}\right\|_{h_{m}}^{2}= & 2^{n} \int_{0}^{\frac{\pi}{2}}\left(\cos \theta_{1}\right)^{2 j_{1}+1}\left(\sin \theta_{1}\right)^{2\left(j_{2}+\cdots+j_{n}+(n-1)-1\right)+1} d \theta_{1} . \\
& \cdot \int_{0}^{\frac{\pi}{2}}\left(\cos \theta_{2}\right)^{2 j_{2}+1}\left(\sin \theta_{2}\right)^{2\left(j_{3}+\cdots+j_{n}+(n-1)-2\right)+1} d \theta_{2} . \\
& \cdot \int_{0}^{\frac{\pi}{2}}\left(\cos \theta_{3}\right)^{2 j_{3}+1}\left(\sin \theta_{3}\right)^{2\left(j_{4}+\cdots+j_{n}+(n-1)-3\right)+1} d \theta_{3} . \\
& \vdots \\
& \cdot \int_{0}^{\frac{\pi}{2}}\left(\cos \theta_{n-2}\right)^{2 j_{n-2}+1}\left(\sin \theta_{n-2}\right)^{2\left(j_{n-1}+j_{n}+(n-1)-(n-2)\right)+1} d \theta_{n-2} . \\
& \cdot \int_{0}^{\frac{\pi}{2}}\left(\cos \theta_{n-1}\right)^{2 j_{n-1}+1}\left(\sin \theta_{n-1}\right)^{2\left(j_{n}+(n-1)-(n-1)\right)+1} d \theta_{n-1} . \\
& \cdot \int_{0}^{+\infty} r^{2\left(j_{1}+\cdots+j_{n}-m\right)+1}\left(1+r^{2}\right)^{n-1} e^{-m r^{2}} d r .
\end{aligned}
$$

For the first $n-1$ integrals, by (6) we find

$$
\begin{aligned}
& \int_{0}^{\frac{\pi}{2}}\left(\cos \theta_{1}\right)^{2 j_{1}+1}\left(\sin \theta_{1}\right)^{2\left(j_{2}+\cdots+j_{n}+(n-1)-1\right)+1} d \theta_{1}=\frac{j_{1} !\left(j_{2}+\cdots+j_{n}+n-2\right) !}{2\left(j_{1}+j_{2}+\cdots+j_{n}+n-1\right) !}, \\
& \int_{0}^{\frac{\pi}{2}}\left(\cos \theta_{2}\right)^{2 j_{2}+1}\left(\sin \theta_{2}\right)^{2\left(j_{3}+\cdots+j_{n}+(n-1)-2\right)+1} d \theta_{2}=\frac{j_{2} !\left(j_{3}+\cdots+j_{n}+n-3\right) !}{2\left(j_{2}+j_{3}+\cdots+j_{n}+n-2\right) !}, \\
& \vdots \\
& \int_{0}^{\frac{\pi}{2}}\left(\cos \theta_{n-2}\right)^{2 j_{n-2}+1}\left(\sin \theta_{n-2}\right)^{2\left(j_{n-1}+j_{n}+(n-1)-(n-2)\right)+1} d \theta_{n-2}=\frac{j_{n-2} !\left(j_{n-1}+j_{n}+1\right) !}{2\left(j_{n-2}+j_{n-1}+j_{n}+2\right) !}, \\
& \int_{0}^{\frac{\pi}{2}}\left(\cos \theta_{n-1}\right)^{2 j_{n-1}+1}\left(\sin \theta_{n-1}\right)^{2\left(j_{n}+(n-1)-(n-1)\right)+1} d \theta_{n-1}=\frac{j_{n-1} ! j_{n} !}{2\left(j_{n-1}+j_{n}+1\right) !} .
\end{aligned}
$$

For the last integral we find

$$
\int_{0}^{+\infty} r^{2\left(j_{1}+\cdots+j_{n}-m\right)+1}\left(1+r^{2}\right)^{n-1} e^{-m r^{2}} d r=\frac{(J-m) !}{2} U(J-m+1, J-m+n+1, m),
$$

where $J=j_{1}+\cdots+j_{n}$ and $U(a, b, z)=\frac{1}{\Gamma(a)} \int_{0}^{\infty} e^{-z t} t^{a-1}(1+t)^{b-a-1} d t$ is the Confluent Hypergeometric Function of the second kind (see [1, page 504]). Since

$$
U(a, b, z)=\frac{\Gamma(1-b)}{\Gamma(a+1-b)}{ }_{1} F_{1}(a, b, z)+\frac{\Gamma(b-1)}{\Gamma(a)} z^{1-b}{ }_{1} F_{1}(a+1-b, 2-b, z),
$$

where ${ }_{1} F_{1}(a, b, z)$ is the Confluent Hypergeometric Function, one gets

$$
\left\|z_{1}^{j_{1}} \cdots z_{n}^{j_{n}}\right\|_{h_{m}}^{2}=m^{m-n-J} \frac{j_{1} ! \cdots j_{n} ! \Gamma(J-m+n)}{\Gamma(J+n)}{ }_{1} F_{1}(1-n, 1+m-n-J, m) .
$$


Therefore

$$
\left\{\frac{z_{1}^{j_{1}} \cdots z_{n}^{j_{n}}}{\left\|z_{1}^{j_{1}} \cdots z_{n}^{j_{n}}\right\|_{h_{m}}}\right\}_{j_{1}+\cdots+j_{m} \geq m}
$$

is a orthonormal basis for the Hilber Space $\left(\mathcal{H}_{m},\langle\cdot, \cdot\rangle_{h_{m}}\right)$. For the function epsilon (setting $J=m+k, k \in \mathbb{N}$ ) follows that

$$
\epsilon_{m g_{S(n)}}(z)=\frac{e^{-m t}}{t^{m}} \sum_{k=0}^{\infty} \frac{m^{k+n} t^{k+m}(k+m+n-1) !}{(k+m) !(k+n-1) !{ }_{1} F_{1}(1-n, 1-k-n, m)},
$$

where $t:=|z|^{2}$. For $n=2,{ }_{1} F_{1}(-1,-1-k, m)=\frac{k+m+1}{k+1}$ and (14) simplifies to $m^{2}$, in agreement with the computations in Section 2. In general, the right-hand side is, in terms of the variable $x:=m t$, equal to

$$
e^{-x} m^{n} \sum_{k=0}^{\infty} \frac{x^{k}(k+m+n-1) !}{(k+m) !(k+n-1) !{ }_{1} F_{1}(1-n, 1-k-n, m)},
$$

which is independent of $x$ only if

$$
\frac{k !(k+m+n-1) !}{(k+m) !(k+n-1) !{ }_{1} F_{1}(1-n, 1-k-n, m)}
$$

is independent of $k$. Looking at the asymptotics as $k \rightarrow+\infty$, the last expression behaves as

$$
1+\frac{m(n-1)(n-2)}{2 k^{2}}+O\left(\frac{1}{k^{3}}\right)
$$

so it can be independent of $k$ only for $n \in\{1,2\}$.

\section{REFERENCES}

[1] Abramowitz, M., Stegun, I. A. Handbook of Mathematical Functions with Formulas, Graphs, and Mathematical Tables. Dover Publications (1972)

[2] Arezzo, C., Loi, A. Quantization of Kähler manifolds and the asymptotic expansion of Tian-Yau-Zelditch. J. Geom. Phys. 47, 87-99 (2003)

[3] Arezzo, C., Loi, A. Moment maps, scalar curvature and quantization of Kähler manifolds. Comm. Math. Phys. 243, 543-559 (2004)

[4] Arezzo, C., Loi, A. Zuddas, F. On homothetic balanced metrics. Ann. Global Anal. Geom. 41, n. 4, 473-491 (2012)

[5] Arezzo, C., Loi, A., Zuddas, F. Szegö kernel, regular quantizations and spherical CRstructures. Math. Z. 275, 1207-1216 (2013)

[6] Arezzo, C., Pacard, F. Blowing up Kähler manifolds with constant scalar curvature. II. Ann. of Math. (2) 170, no. 2, 685-738 (2009)

[7] Berezin, F. A. Quantization. Math. USSR Izvestiya 8, 1109-1163 (1974)

[8] Bi, E., Feng, Z., Tu, Z. Balanced metrics on the Fock-Bargmann-Hartogs domains. Ann. Glob. Anal. Geom. 49, 349-359 (2016)

[9] Cahen, M., Gutt, S., Rawnsley, J. H. Quantization of Kähler manifolds I: geometric interpretation of Berezin's quantization. J. Geom. Phys. 7, 45-62 (1990)

[10] Calabi, E. Isometric imbeddings of complex manifolds. Ann. Math. 58, 1-23 (1953) 
[11] Cannas Aghedu, F. On the balanced condition for the Eguchi-Hanson metric, J. Geom. Phys. 137, 35-39 (2019)

[12] Di Scala, A., Ishi, H., Loi, A. Kähler Immersions of Homogeneous Kähler Manifolds into Complex Space Forms. Asian J. Math. 16 (3), 479-488 (2012)

[13] Donaldson, S. Scalar curvature and projective embeddings. I. J. Diff. Geom. 59, 479$522(2001)$

[14] Engliš, M. Berezin quantization and reproducing kernels on complex domains. Trans. Am. Math. Soc. 348, 411-479 (1996)

[15] Engliš, M. Weighted Bergman kernels and balanced metrics. RIMS Kokyuroku 1487, 40-54 (2006)

[16] Feng Z., Tu Z., On canonical metrics on Cartan-Hartogs domains, Math. Zeit. 278, Issue 1-2, 301-320 (2014)

[17] Greco, A., Loi, A. Radial balanced metrics on the unit disk. J. Geom. Phys. 60, 53-59 (2010)

[18] Griffiths, P., Harris, J. Principles of Algebraic Geometry. John Wiley \& Sons, Inc. (1978)

[19] Loi, A. The Tian-Yau-Zelditch asymptotic expansion for real analytic Kähler metrics. Int. J. Geom. Methods Mod. Phys. 1, 253-263 (2004)

[20] Loi, A. A Laplace integral, the T-Y-Z expansion and Berezin's transform on a Kähler manifold. Int. J. Geom. Meth. Mod. Phys. 2, 359-371 (2005)

[21] Loi, A. Regular quantizations of Kähler manifolds and constant scalar curvature metrics. J. Geom. Phys. 53 (3), 354-364 (2005)

[22] Loi, A. Calabi's diastasis function for Hermitian symmetric spaces. Diff. Geom.Appl. 24, 311-319 (2006)

[23] Loi, A., Mossa, R. Berezin quantization of homogeneous bounded domains. Geom. Dedicata 161, 119-128 (2012)

[24] Loi, A., Mossa, R. Some remarks on Homogeneous Kähler manifolds. Geom. Dedicata Volume 179, 1-7 (2015)

[25] Loi, A., Mossa, R., Zuddas, F. The log-term of the disc bundle over a homogeneous Hodge manifold. Ann. Global Anal. Geom. 51 no. 1, 35-51 (2017)

[26] Loi, A., Salis, F., Zuddas, F. Two conjectures on Ricci-flat Kähler metrics. Math. Zeit. $1,1-15(2018)$

[27] Loi, A., Zedda, M. Balanced metrics on Cartan and Cartan-Hartogs domains. Math. Z. 270, no. 3-4, 1077-1087 (2012)

[28] Loi, A., Zedda, M., Zuddas, F. Some remarks on the Kähler geometry of the TaubNUT metrics. Ann. Global Anal. Geom. 41, no. 4, 515-533 (2012)

[29] Loi, A., Zedda, M. On the coefficients of TYZ expansion of locally Hermitian symmetric spaces. Manuscripta math. 148, 303-315 (2015)

[30] Lu, Z. On the lower terms of the asymptotic expansion of Tian-Yau-Zelditch. Am. J. Math. 122, 235-273 (2000)

[31] Lu, Z., Tian, G. The log term of Szegö Kernel. Duke Math. J. Volume 125, No 2 351-387 (2004)

[32] McDuff, D., Salamon, D. Introduction to Symplectic Topology. Oxford (2017)

[33] Rawnsley, J. Coherent states and Kähler manifolds. Q. J. Math. Oxford (2) 28, 403-415 (1977) 
[34] Simanca, Santiago R. Kähler metrics of constant scalar curvature on bundles over $\mathbb{C} P^{n-1}$. Math. Ann. 291, 239-246 (1991)

[35] $\mathrm{Xu}, \mathrm{H}$. A closed formula for the asymptotic expansion of the Bergman kernel. Commun. Math. Phys. 314(3), 555-585 (2012)

[36] Zedda M. Canonical metrics on Cartan-Hartogs domains. Int. J. Geom. Methods Mod. Phys. 9 , no. 1. (2012)

[37] Zelditch, S. Szegö Kernels and a Theorem of Tian. Int. Math. Res. Not. 6, 317-331 (1998)

Dipartimento di Matematica e Informatica, Università di Cagliari, Via Ospedale 72, 09124 CAGLIARI (ITALY)

E-mail address: fcannasaghedu@unica.it, loi@unica.it 